\title{
Extended General Relativity for a Curved Universe
}

\author{
Mohammed. B. Al-Fadhli ${ }^{1 *}$
}

${ }^{1}$ College of Science, University of Lincoln, Lincoln, LN6 7TS, UK.

\begin{abstract}
The recent Planck Legacy release revealed the presence of an enhanced lensing amplitude in the cosmic microwave background (CMB). Notably, this amplitude is higher than that estimated by the lambda cold dark matter model $(\Lambda \mathrm{CDM})$, which endorses the positive curvature of the early Universe with a confidence level greater than $99 \%$. Although General Relativity (GR) performs accurately in the local/present Universe where spacetime is almost flat, its lost boundary term, incompatibility with quantum mechanics and the necessity of dark matter and dark energy might indicate its incompleteness. By utilising the Einstein-Hilbert action, this study presents extended field equations considering the pre-existing/background curvature and the boundary contribution. The extended field equations consist of Einstein field equations with a conformal transformation feature in addition to the boundary term, which could remove singularities from the theory and facilitate its quantisation. The extended equations have been utilised to derive the evolution of the Universe with reference to the scale factor of the early Universe and its radius of curvature.
\end{abstract}

Keywords: Astrometry, General Relativity, Boundary Term.

\section{INTRODUCTION}

Considerable efforts have been devoted to modifying gravity in form of Conformal Gravity, Loop Quantum Gravity, MOND, ADS-CFT, String theory, $F(R)$ Gravity, etc. (Mannheim, 1997, 2001; Randall and Sundrum, 1999; Garriga and Tanaka, 1999; Germani and Sopuerta, 2002; Nojiri and Odintsov, 2006, 2010; Amendola et al., 2007; Appleby and Battye, 2007; Bekenstein, 2007; Nelson, 2010).

Motivation to modify General Relativity (GR) aimed to elucidate possible existence or the form of dark matter/energy, achieve a better description of observation data, verify theoretical restrictions in the strong curvature regime such as within back holes as well as to formulate Quantum Gravity (Appleby and Battye, 2007; Nelson, 2010). To achieve an efficient action for quantum corrections, several theories have been formulated on the modification of Lagrangian gravitational fields. Such modifications appear to be inevitable, which included higher-order curvature terms as well as non-minimally coupled scalar fields (Vilkovisky, 1992; Capozziello and Lambiase, 2000; Garattini, 2013). However, these modifications have to be consistent with the energy conservation law.

*E-mail: $\underline{\text { malfadhli@lincoln.ac.uk }}$
Recent evidence by Planck Legacy recent release (PL18) indicated the presence of an enhanced lensing amplitude in the cosmic microwave background $(\mathrm{CMB})$, which is notably higher than that estimated by the lambda cold dark matter model $(\Lambda C D M)$. This endorses the positive curvature of the early Universe with a confidence level greater than 99\% (Aghanim et al., 2020; Di Valentino, Melchiorri and Silk, 2020). Besides, the gravitational lensing by substructures of several galaxy clusters is an order of magnitude more than the $\Lambda \mathrm{CDM}$ estimation (Meneghetti et al., 2020; Umetsu, 2020). This evidence endorses a spatially curved Universe in spite of the spacetime flatness of the local/present Universe. Further, a closed Universe can provide an agreement with the observed $\mathrm{CMB}$ anisotropy (Efstathiou, 2003).

Accordingly, in this study, the background/preexisting curvature has been incorporated into the Einstein-Hilbert action. However, to comply with the energy conservation law and the compatibility of the action, a new modulus of deformation/curvature of spacetime continuum is utilised, which is formulated on the theory of elasticity (Landau, 1986).

The paper is organised as follows. Section 2 discusses the origination of the spacetime continuum modulus of curvature and mathematical derivations of the extended field equations. Sections 3 discusses the derivations of referenced Friedmann equations and boundary contributions. Section 4 summarises the outcomes and conclusions. Section 5 suggests the future development of this work. 


\section{Extended Field Equations}

To consider pre-existing/background curvatures signified by the scalar curvature $\mathcal{R}$, a new modulus of deformation/curvature of the spacetime continuum $E_{D}=$ (stress/strain) in $\left(\mathrm{N} / \mathrm{m}^{2}\right)$ is defined, which can be expressed using Einstein field equations as

$E_{D}=\frac{T_{\mu}^{v}-T \delta_{\mu}^{v} / 2}{R_{\mu}^{v} / \mathcal{R}}=\mathcal{R} \frac{\mathrm{c}^{4}}{8 \pi \mathrm{G}}$

where the stress is signified by the stress-energy tensor $T_{v}^{\mu}$ of trace $T$, while the strain is signified by the Ricci curvature tensor $R_{v}^{\mu}$ as the change in the curvature divided by pre-existing curvature $\mathcal{R}$. $\delta_{u}^{v}$ is the Kronecker delta (Straumann, 2013). According to the theory of elasticity, the modulus times the volume equals internal energy of reversible systems (Landau, 1986). Thus, $E_{D}$ could represent the internal energy density of the space, i.e., the vacuum energy density. $E_{D}$ is proportional to the fourth-order of the speed of light, resembling Quantum field theory (QFT) energy cut-off predictions of vacuum energy density (Rugh and Zinkernagel, 2000). According to conservation of energy law, the Einstein-Hilbert action is extended to

$S=\int\left[T+\mathcal{L}_{M}\right] \sqrt{-g} d^{4} x=\int\left[\frac{E_{D}}{2} \frac{R}{\mathcal{R}}+\mathcal{L}_{M}\right] \sqrt{-g} d^{4} x$

where $T$ is the kinetic term, $\mathcal{L}_{M}$ is the Lagrangian matter density denoting the potential term, $R$ is the Ricci scalar curvature and $g$ is the determinant of the metric $g_{u v}$. According to the principle of least action, Eq. (2) should hold for any variation in the metric as

$\delta S=\int\left[\frac{E_{D}}{2}\left(\frac{\delta\left(R \mathcal{R}^{-1} \sqrt{-g}\right)}{\delta g^{\mu \nu}}\right)+\frac{\delta\left(\mathcal{L}_{M} \sqrt{-g}\right)}{\delta g^{\mu \nu}}\right] \delta g^{\mu \nu} d^{4} x$

The differentiation yields

$$
\begin{aligned}
\delta S=\int\left[\frac { E _ { D } } { 2 } \left(\frac{\delta R \sqrt{-g}}{\mathcal{R} \delta g^{\mu \nu}}\right.\right. & \left.-\frac{\delta \mathcal{R} \sqrt{-g} R}{\mathcal{R}^{2} \delta g^{\mu \nu}}+\frac{\delta \sqrt{-g} R}{\mathcal{R} \delta g^{\mu \nu}}\right) \\
& \left.+\frac{\delta \sqrt{-g} \mathcal{L}_{M}}{\delta g^{\mu \nu}}+\frac{\delta \mathcal{L}_{M} \sqrt{-g}}{\delta g^{\mu \nu}}\right] \delta g^{\mu \nu} d^{4} x
\end{aligned}
$$

By performing the differentiation of the determinant according to the Jacobi's formula as $\delta g=g g^{\mu v} \delta g_{\mu \nu}$, $\delta \sqrt{-g}=-\delta g / 2 \sqrt{-g}$ while $\delta g^{\mu v} g_{\mu \nu}=-\delta g_{\mu \nu} g^{\mu \nu}$ (S. M. Carroll, 2003) in addition to the differentiation of the scalar curvature $R$ which equals $R_{\mu \nu} g^{\mu \nu}$ as

$$
\delta S=\int\left[\begin{array}{c}
\frac{R_{\mu v} \delta g^{\mu v}+g^{u v} \delta R_{\mu v}}{\mathcal{R} \delta g^{\mu v}} \\
\frac{E_{D}}{2}\left(\begin{array}{c}
\mathcal{R}_{\mu \nu} \delta g^{\mu \nu}+g^{\mu v} \delta \mathcal{R}_{\mu v} \\
\mathcal{R}^{2} \delta g^{\mu \nu}
\end{array}\right. \\
-\frac{g_{\mu v}}{2 \mathcal{R}} R \\
-\frac{g_{\mu v}}{2} \mathcal{L}_{M}+\frac{\delta \mathcal{L}_{M}}{\delta g^{\mu \nu}}
\end{array}\right] \sqrt{-g} \delta g^{\mu v} d^{4} x
$$

The lambda/pressure is not considered which might be incorporated implicitly into stress-energy tensors.
By extracting one boundary part from Eq. (5) as

$\int g^{\mu v} \delta R_{\mu v} \sqrt{-g} d^{4} x$

The variation in the Ricci curvature tensor $\delta R_{u v}$ can be written in terms of the covariant derivative of the difference between two Levi-Civita connections, the Palatini identity:

$\delta R_{\mu \nu}=\nabla_{\rho}\left(\delta \Gamma_{\mu \nu}^{\rho}\right)-\nabla_{\nu}\left(\delta \Gamma_{\mu \rho}^{\rho}\right)$

The Ricci curvature tensor variation with respect to the inverse metric tensor $g^{\mu v}$ can be obtained utilising the metric compatibility of the covariant derivative, $\nabla_{\rho} g^{\mu \nu}=0$ (S. M. Carroll, 2003) as

$g^{\mu v} \delta R_{\mu \nu}=\nabla_{\rho}\left(g^{\mu v} \delta \Gamma_{\mu \nu}^{\rho}\right)-\nabla_{v}\left(g^{\mu v} \delta \Gamma_{\mu \rho}^{\rho}\right)$

Thus, the boundary part as a total derivative for any tensor density can be transformed according to the Stokes' theorem with renaming dummy indices as

$$
\begin{gathered}
\iiint_{V} \nabla_{\mu}\left(g^{\sigma v} \delta \Gamma_{v \sigma}^{\mu}-g^{\sigma \mu} \delta \Gamma_{\mu \sigma}^{\mu}\right) \sqrt{-g} d V \equiv \iiint_{V} \nabla_{\mu} A^{\mu} \sqrt{-g} d V \\
=\oiint_{S} A^{\mu} \cdot \hat{n}_{u} \sqrt{-g} d S=\oint_{\partial V} K \epsilon \sqrt{|\mathrm{q}|} d^{3} x
\end{gathered}
$$

where $K=K_{u v} q^{\mu v}$ is the trace of extrinsic curvature as the second fundamental form, $q$ is the determinant of the induced metric on the manifold boundary, and $\epsilon$ equals 1 when the normal $\hat{n}_{u}$ on the boundary is a spacelike entity and equals -1 when the normal is a timelike entity (Dyer and Hinterbichler, 2009). Thus, the action in Eq. (2) can be rewritten in parts as

$$
\begin{aligned}
S_{c}+S_{b}+S_{m}=\int \frac{E_{D}}{2 \mathcal{R}}\left(R_{c} \sqrt{-g} d^{4} x\right. & \left.+K \epsilon \sqrt{|q|} d^{3} x\right) \\
& +\int \mathcal{L}_{M} \sqrt{-g} d^{4} x
\end{aligned}
$$

where $S_{c}, S_{b}$, and $S_{m}$ denote the contributions to the action from the curvature without the boundary, the boundary alone and the matter fields respectively.

To solve the parted action, firstly, the action without boundary parts is reduced to the following

$$
\begin{gathered}
\delta S_{c}+\delta S_{m}= \\
\int\left[\begin{array}{c}
\frac{E_{D}}{2}\left(\frac{R_{\mu \nu} \delta g^{\mu \nu}}{\mathcal{R} \delta g^{\mu \nu}}-\frac{\mathcal{R}_{\mu \nu} \delta g^{\mu \nu}}{\mathcal{R}^{2} \delta g^{\mu \nu}} R-\frac{g_{\mu \nu}}{2 \mathcal{R}} R\right) \\
-\frac{g_{\mu \nu}}{2} \mathcal{L}_{M}+\frac{\delta \mathcal{L}_{M}}{\delta g^{\mu \nu}}
\end{array}\right] \sqrt{-g} \delta g^{\mu \nu} d^{4} x
\end{gathered}
$$

The stress energy-momentum tensor is proportional to the Lagrangian term in the action by the definition (Straumann, 2013) as

$T_{u v}:=g_{\mu \nu} \mathcal{L}_{M}-\frac{2 \delta \mathcal{L}_{M}}{\delta g^{\mu \nu}}$

Additionally, the energy-momentum tensor includes pressure terms according to the equation of state (Vikman, 2005). 
By substituting Eqs. $(1,12)$ in Eq. $(11)$ and using the principle of least action yield

$R_{\mu \nu}-\frac{\mathcal{R}_{\mu \nu}}{\mathcal{R}} R-\frac{1}{2} R g_{\mu \nu}=\frac{8 \pi G}{c^{4}} T_{\mu \nu}$

here $\mathcal{R}_{\mu \nu} / \mathcal{R}=\mathcal{R}_{\mu \nu} / \mathcal{R}_{\mu \nu} \bar{g}^{\mu \nu}=\bar{g}_{\mu \nu}$ corresponds to Weyl's conformal transformation of the metric (Kozameh et al., 1985; Straub, 2006). Eq. (13) can be rewritten as

$R_{\mu \nu}-\frac{1}{2} R \hat{g}_{\mu \nu}=\frac{8 \pi G}{c^{4}} T_{\mu \nu}$

where $\hat{g}_{\mu \nu}=g_{\mu \nu}+2 \bar{g}_{\mu \nu}$ is the conformal transformation of the metric tensor due to the fact that Einstein spaces are a subclass of conformal spaces (Kozameh et al., 1985). The conformal transformation of Einstein field equations could describe the tidal distortion of gravitational waves in absence of matter (Penrose, 2005) and the galaxy rotation curve as they account for the Universe curvature evolution over its age or over the conformal time.

Secondly, concerning the boundary action, the variation in the boundary part $K \epsilon \sqrt{|q|} d^{3} x$ in respect to its inverse induced metric $q^{\mu \nu}$ is

$\int\left[\left(\frac{K_{\mu \nu} \delta q^{\mu \nu}+q^{\mu \nu} \delta K_{\mu \nu}}{\delta q^{\mu \nu}}-K \frac{q_{\mu \nu}}{2}\right) \epsilon\right] \sqrt{|q|} \delta q^{\mu v} d^{3} x$

as $q^{\mu \nu}=-q_{\mu \nu} \delta q^{\mu \nu} / \delta q_{\mu \nu}$, Eq. (15) can be written as

$\int\left[\left(\frac{K_{\mu \nu} \delta q^{\mu \nu}}{\delta q^{\mu \nu}}-\frac{K}{2}\left(q_{\mu \nu}+2 q_{\mu \nu} \frac{\delta K_{\mu \nu}}{\delta q_{\mu \nu} K}\right)\right) \epsilon\right] \sqrt{|q|} \delta q^{\mu v} d^{3} x$

where $\delta K_{\mu \nu} / \delta q_{\mu \nu} K$ could can resemble the conformal distortion of the boundary curvature as with Eq.(13) corresponding the Weyl's conformal transformation, which can be expressed as a positive function $\Omega^{2}$ on the spacetime boundary manifold as follows

$\int\left[\left(\frac{K_{\mu \nu} \delta q^{\mu \nu}}{\delta q^{\mu \nu}}-\frac{K}{2}\left(q_{\mu \nu}+2 q_{\mu \nu} \Omega^{2}\right)\right) \epsilon\right] \sqrt{|q|} \delta q^{\mu \nu} d^{3} x$

By substituting the variation in the boundary part in Eq. (17) into the full action in Eq. (5) according to Eq. (10), the variation in boundary action is

$$
\begin{aligned}
& \delta S_{b}= \\
& \left.\int\left[\begin{array}{c}
\frac{K_{\mu \nu} \delta q^{\mu \nu}}{\mathcal{R} \delta q^{\mu \nu}}-\frac{K}{2 \mathcal{R}}\left(q_{\mu \nu}+2 \bar{q}_{\mu \nu}\right) \\
\frac{-K_{\mu \nu} \delta q^{\mu \nu} R}{\mathcal{R}^{2} \delta q^{\mu \nu}}+\frac{K R}{2 \mathcal{R}^{2}}\left(q_{\mu \nu}+2 \bar{q}_{\mu \nu}\right)
\end{array}\right) \epsilon\right] \sqrt{|q|} \delta q^{\mu \nu} d^{3} x
\end{aligned}
$$

where $\bar{q}_{\mu \nu}$ is the conformal induced metric $q_{\mu \nu} \Omega^{2}$. By chosen $\epsilon$ as a timelike entity, substituting Eq. (1) in Eq. (18) and using the principle of least action

$-K_{\mu \nu}+\frac{1}{2} K \hat{q}_{\mu \nu}+\frac{R}{\mathcal{R}} K_{\mu \nu}-\frac{1}{2} \frac{R}{\mathcal{R}} K \hat{q}_{\mu \nu}$

where $\hat{q}_{\mu v}$ is the conformally transformed induced metric on the spacetime manifold boundary.
By combining action parts in Eqs. $(13,19)$ according to Eq. (10), the new extended field equations are

$R_{\mu \nu}-\frac{1}{2} R \hat{g}_{\mu \nu}+\frac{R-\mathcal{R}}{\mathcal{R}}\left(K_{\mu \nu}-\frac{1}{2} K \hat{q}_{\mu \nu}\right)=\frac{8 \pi G}{c^{4}} T_{\mu \nu}$

The boundary tensor/term is only significant at highenergy limits such as with black holes (Dyer and Hinterbichler, 2009) and the early Universe, and can remove the singularities from the theory.

\section{Universe Evolution Model}

The Friedmann-Lemaîtree-Robertson-Walker (FLRW) metric model is the standard cosmological model, which assumes an isotropic and homogenous Universe (Ellis and van Elst, 1998; Lachì Eze-Rey and Luminet, 2003), where the isotropy and homogeneity of the early Universe based on the CMB are consistent with this model.

\subsection{Referenced FLRW Metric Model}

According to the PL18 release which revealed the positive curvature of a closed early Universe (Aghanim et al., 2020; Di Valentino, Melchiorri and Silk, 2020), the reference radius of curvature $r_{P}$ upon the emission of the $\mathrm{CMB}$ representing the early Universe curvature radius and the corresponding early Universe scale factor $a_{P}$ at the reference time $t_{p}$ are incorporated to reference the FLRW metric model as shown in Figure 1.

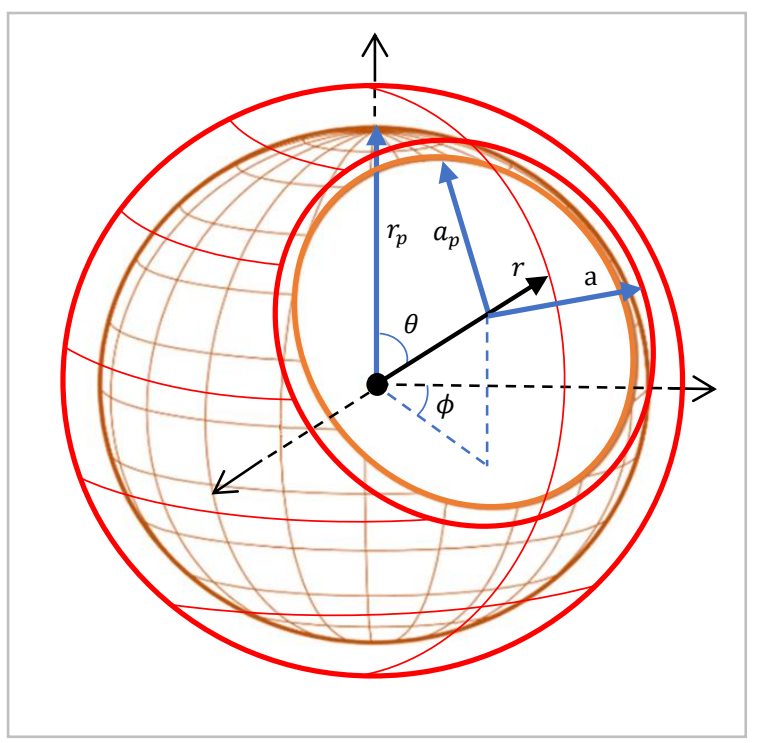

Figure 1. The hypersphere of a positively curved early Universe expansion upon the CMB emissions. $r_{p}$ is the reference radius of the intrinsic curvature and $a_{p}$ is the reference scale factor of the early Universe at the corresponding reference time $t_{p} \cdot a / a_{P}$ denotes a new dimensionless scale factor of this referenced metric. $r, \phi, \theta$ are the comoving coordinates. 
The four-dimensional spacetime interval $d s$ of the referenced metric is

$$
\begin{gathered}
d s^{2}=c^{2} d t^{2}-\frac{a^{2}}{a_{p}^{2}}\left(\frac{d r^{2}}{1-\left(r^{2} / r_{p}^{2}\right)}+r^{2} d \theta^{2}\right. \\
\left.+r^{2} \sin ^{2} \theta d \phi^{2}\right)
\end{gathered}
$$

where $a / a_{p}$ is the new dimensionless scale factor and $r, \phi, \theta$ are the comoving coordinates. Accordingly, the metric components are

$g_{t t}=c^{2}, \quad g_{r r}=-\left(\frac{a^{2}}{a_{p}^{2}}\right) /\left(1-\frac{r^{2}}{r_{p}^{2}}\right)$,

$g_{\theta \theta}=-\left(\frac{a^{2}}{a_{p}^{2}}\right) r^{2}, \quad g_{\phi \phi}=-\left(\frac{a^{2}}{a_{p}^{2}}\right) r^{2} \sin ^{2} \theta$.

The Ricci curvature tensor $R_{u v}$ is solved using Christoffel symbols for $g_{u v}$ in Eqs. (22) as well as the Ricci scalar curvature $R$ as follows

$R_{r r}=\frac{1}{c^{2}}\left(\frac{a \ddot{a}}{a_{p}^{2}}+\frac{2 \dot{a}^{2}}{a_{p}^{2}}+\frac{2 c^{2}}{r_{p}^{2}}\right) /\left(1-\frac{r^{2}}{r_{p}^{2}}\right)$,

$R_{\theta \theta}=\frac{r^{2}}{c^{2}}\left(\frac{a \ddot{a}}{a_{p}^{2}}+\frac{2 \dot{a}^{2}}{a_{p}^{2}}+\frac{2 c^{2}}{r_{p}^{2}}\right)$,

$R_{\phi \phi}=\frac{r^{2} \sin ^{2} \theta}{c^{2}}\left(\frac{a \ddot{a}}{a_{p}^{2}}+\frac{2 \dot{a}^{2}}{a_{p}^{2}}+\frac{2 c^{2}}{r_{p}^{2}}\right)$,

$R_{t t}=-3 \frac{\ddot{a}}{a}, R=\frac{-6}{c^{2}}\left(\frac{\ddot{a}}{a}+\frac{\dot{a}^{2}}{a^{2}}+\frac{c^{2} a_{p}^{2}}{a^{2} r_{p}^{2}}\right)$.

The derivations are presented in Appendix A. No conformal transformation is applied for the metric thus, its outcomes are comparable with literature. Besides Appendix B presents well-defined Christoffel symbols of the conformally transformed metric $\hat{g}_{\mu \nu}$.

\subsection{Referenced Friedmann Equations}

By solving the field equations for a perfect fluid given by $T_{\mu \nu}=\left(\rho+P / c^{2}\right) u_{\mu} u_{v}+P g_{\mu \nu}$ (Straumann, 2013) and substituting Eqs. (22,23), Friedmann equations counting for the reference curvature radius and the reference scale factor are

$H^{2} \equiv \frac{\dot{a}^{2}}{a^{2}}=\frac{8 \pi G \rho}{3}-\frac{c^{2} a_{p}^{2}}{a^{2} r_{p}^{2}}$,

$\dot{H} \equiv \frac{\ddot{a}}{a}=-\frac{4 \pi G}{3}\left(\rho+3 \frac{P}{c^{2}}\right)$.

where $H, P$, and $\rho$ are the Hubble parameter, pressure, and density respectively. The equations do not show a conformal distortion. By utilising the imaginary time $\tau=i t$, the referenced Friedmann equations can be solved by rewriting Eq. (24) in terms of the conformal time in its parametric form $d \eta=$ $-i \frac{a_{p}}{a} d \tau$ at the reference imaginary time $\tau_{p}$ and the corresponding reference scale factor $a_{p}$ as

$\int_{0}^{\eta} d \eta=\int_{0}^{2 \pi} a_{p}\left(\frac{8 \pi G \rho_{p} a_{p}^{3}}{3} a-\frac{c^{2} a_{p}^{2}}{r_{p}^{2}} a^{2}\right)^{-1 / 2} d a$

where $\rho=\rho_{p} a_{p}{ }^{3} / a^{3}$ (Ryden, 2006).
By integrating, the scale factor evolution is

$a(\eta) / a_{p}=\frac{M_{p} G}{c^{2} r_{p}}\left(1-\cos \frac{c}{r_{p}} \eta\right)$

where $M_{p}=\frac{4}{3} \pi \rho_{p} r_{p}{ }^{3}$ is the mass of the early Universe plasma. The constant in Eq. (27) can be written in terms of the modulus $E_{D}$ representing the vacuum energy density and the Universe energy density $E$ using Eq. (1) as $E / 6 E_{D}$.

Additionally, the evolution of the imaginary time $\tau(\eta)$ can be obtained by integrating the spatial factor over the expansion speed $H_{\eta}$ while initiating at the reference imaginary time $\tau_{p}$ and the corresponding spatial factor $a_{p}$. Thus, by rewriting Eq. (27) in terms of the Hubble parameter by its definition at $\tau_{p}$ as $d \tau=i \frac{d a}{H a_{p}}$ as

$\int_{\tau_{P}}^{\tau} d \tau=i \int_{0}^{\eta} \frac{E}{6 H_{\eta} E_{D}}\left(1-\cos \frac{c}{r_{p}} \eta\right) d \eta$

By integration, the imaginary time evolution is

$\tau(\eta)=i \frac{E}{6 H_{\eta} E_{D}}\left(\eta-\sin \frac{c}{r_{0}} \eta\right)+\tau_{p}$

where $\tau_{p}$ denotes the reference imaginary time.

According to the energy conservation law and because $\rho a^{3}=\rho_{p} a_{p}{ }^{3}=$ constant (Ryden, 2006) and by substituting the spatial scale factor rate in Eq. (27) to $\rho a^{3}=$ constant, the evolution of density is

$\rho_{\eta}=D_{p}\left(1-\cos \frac{c}{r_{p}} \eta\right)^{-3}$

where $D_{P}$ is a constant. According to Eq. (25), the Hubble parameter is dependent on the density; thus, by substituting Eq. (30) to Eq. (25) and initiating the integration at $\tau_{p}$, thus, $\dot{H}=\frac{\ddot{a}}{a_{p}}$ as

$\int_{H_{p}}^{H} \dot{H}=\int_{0}^{\eta} \frac{-4 \pi G D_{p}}{3}\left(1-\cos \frac{c}{r_{p}} \eta\right)^{-3} d \eta$

By integration, the Hubble parameter evolution is

$H_{\eta}=H_{a}\left(\frac{1}{5} \cot ^{5} \frac{c}{2 r_{p}} \eta+\frac{2}{3} \cot ^{3} \frac{c}{2 r_{p}} \eta+\cot \frac{c}{2 r_{p}} \eta\right)+H_{p}$

where, $H_{a}$ and $H_{p}$ are the integration constants. By combining Eqs. $(27,29-32)$ in complex plan results in the hyper-spherical spacetime wave function with respect the reference curvature radius as

$$
\begin{aligned}
\underset{0<\eta \leq 2 \pi}{\overrightarrow{\psi_{L}}(\eta) / r_{p}} & =\mp \frac{E}{6 E_{D}}\left(\left(1-\cos \frac{c}{r_{p}} \eta\right)^{2}\right. \\
& \left.+\frac{c^{2}}{H_{\eta}^{2} a_{p}^{2}}\left(\eta-\sin \frac{c}{r_{p}} \eta\right)^{2}\right)^{1 / 2} e^{i \cot \frac{H_{\eta} a_{p}\left(1-\cos \frac{c}{r_{p}} \eta\right)}{c\left(\eta-\sin \frac{c}{r_{p}} \eta\right)}}
\end{aligned}
$$

where $E / E_{D}$ is a dimensionless energy parameter as the ratio of the Universe energy density to the vacuum energy density. The positive and negative solutions of the wave function imply the evolution in two directions. 


\subsection{Early Universe Boundary Contribution}

For high energy limits, gravitational contributions of early Universe plasma boundary can be obtained using the boundary term in the extended field equations in Eqs. (20); at the reference imaginary time $\tau_{p}$, there is no conformal transformation. Therefore, the induced metric tensor on early Universe plasma hypersphere $q_{\mu v}$ is given in Eqs. (34), where $R$ is the extrinsic curvature radius. The extrinsic curvature tensor is solved utilising the formula $K_{u v}=-\overrightarrow{T_{\mu}} \cdot \nabla_{v} \overrightarrow{N_{v}}$. Due to the hypersphere similarity, the covariant derivative reduces to the partial derivative as $K_{u v}=$ $-\overrightarrow{T_{\mu}} \partial \vec{N} / \partial \overrightarrow{T^{v}}$ (Pavel Grinfeld, 2013) as

$$
\begin{aligned}
q_{\mu \nu} & =\left(\begin{array}{ccc}
\mathrm{c}^{2} & 0 & 0 \\
0 & \frac{a^{2}}{a_{P}{ }^{2}} R^{2} & 0 \\
0 & 0 & \frac{a^{2}}{a_{P}{ }^{2}} R^{2} \sin ^{2} \theta
\end{array}\right), \\
K_{\mu \nu} & =\left(\begin{array}{ccc}
0 & 0 & 0 \\
0 & -\frac{a^{2}}{a_{P}{ }^{2}} R & 0 \\
0 & 0 & -\frac{a^{2}}{a_{P}{ }^{2}} R \sin ^{2} \theta
\end{array}\right) .
\end{aligned}
$$

The trace of the extrinsic curvature is $K=K_{\mu \nu} q^{\mu \nu}=$ $-2 / R$. The pre-existing curvature of early Universe plasma boundary at the reference imaginary time $\tau_{p}$ is the Gaussian curvature $\mathcal{R}_{p}=1 / r_{p}^{2}$ (Pavel Grinfeld, 2013).

On the other hand, the Ricci scalar curvature $R$ at $\tau_{p}$ can be written as the difference between kinetic and potential energy densities whereby substituting Friedmann equations in Eqs. $(24,25)$ into the Ricci scalar curvature $R$ in Eqs. (23) as

$R_{p}=\frac{6 G_{p}}{c^{2}}\left(\frac{4 \pi P_{p}}{c^{2}}-\frac{4 \pi \rho_{p}}{3}\right)$

By solving the boundary term $\frac{\mathcal{R}-R}{\mathcal{R}}\left(K_{\mu \nu}-\right.$ $\left.\frac{1}{2} K q_{\mu \nu}\right)=\frac{8 \pi G_{p}}{c^{4}} T_{\mu \nu}$ for a perfect fluid given by $T_{\mu \nu}=(\rho+$ $\left.\frac{P}{c^{2}}\right) u_{\mu} u_{v}+P g_{\mu \nu}$ (Straumann, 2013) whereas the normal on the boundary is chosen as a spacelike entity, and then substituting Eqs. (35-34) into the boundary term as follows

$\frac{\frac{1}{r_{p}^{2}}-\frac{6 G_{p}}{c^{2}}\left(\frac{4 \pi P_{p}}{c^{2}}-\frac{4 \pi \rho_{p}}{3}\right)}{1 / r_{p}^{2}}\left(\frac{\mathrm{c}^{2}}{r_{p}^{2}}\right)=8 \pi G_{p} \rho_{p}$

Multiplying by early Universe volume $V_{p}$ yields

$r_{p}=\frac{4 G_{p} P_{p} V_{p}}{c^{4}}$

The reference curvature radius $r_{p}>0$ because any reduction in the volume causes an increase in the pressure.

\section{Conclusions}

In this study, pre-existing universal curvatures and boundary contributions were considered to derive the extended field equations using the Einstein -Hilbert action. The extended field equations consist of Einstein field equations corresponding to Weyl's conformal transformation of the metric in addition to the boundary term, which removes singularities from the theory.

The early Universe was modelled utilising the referenced FLRW metric model. The extended field equations were used to derive the evolution of the Universe in reference with the scale factor of the early Universe and its radius of curvature. The positive and negative solutions of the wave function might imply the evolution in two directions. The derived Hubble evolution shows acceleration/deceleration. This may resolve Hubble tension while the reference radius of curvature might be the smallest possible radius of the early Universe due to the boundary contribution.

\section{Future Work}

The conformal metric tensor will be to utilised investigate the conformal distortion of the Friedmann equations.

Acknowledgement: I am grateful to the Preprints Editors Ms Mila Marinkovic and Ms Bojana Djokic for their rapid and excellent attention in the processing of the submissions.

Conflicts of Interest: The author declares no conflict of interest.

Funding: This research received no funding.

\section{Appendix A}

The Ricci curvature tensor $R_{u v}$ is solved using the Christoffel symbols of the second kind given by $\Gamma_{\mu \nu}^{\rho}=\frac{1}{2} g^{\rho \lambda}\left(\partial_{\mu} g_{\lambda \nu}+\partial_{\nu} g_{\lambda \mu}-\partial_{\lambda} g_{\mu \nu}\right)$ for the referenced

\begin{tabular}{|c|c|}
\hline$\Gamma_{11}^{0}=\frac{a \dot{a}}{c^{2} a_{p}{ }^{2}\left(1-\frac{r^{2}}{r_{p}^{2}}\right)}$ & $\Gamma_{11}^{1}=\frac{r}{r_{p}^{2}\left(1-\frac{r^{2}}{r_{p}^{2}}\right)}$ \\
\hline$\Gamma_{22}^{0}=\frac{r^{2} a \dot{a}}{c^{2} a_{p}^{2}}$ & $\Gamma_{22}^{1}=-\mathrm{r}\left(1-\frac{r^{2}}{r_{p}^{2}}\right)$ \\
\hline$\Gamma_{33}^{0}=\frac{r^{2} a \dot{a} \sin ^{2} \theta}{c^{2} a_{p}{ }^{2}}$ & $\Gamma_{33}^{1}=-r \sin ^{2} \theta\left(1-\frac{r^{2}}{r_{p}^{2}}\right)$ \\
\hline \multicolumn{2}{|c|}{$\Gamma_{01}^{1}=\Gamma_{02}^{2}=\Gamma_{03}^{3}=\Gamma_{10}^{1}=\Gamma_{20}^{2}=\Gamma_{30}^{3}=\frac{\dot{a}}{a}$} \\
\hline \multicolumn{2}{|c|}{$\Gamma_{12}^{2}=\Gamma_{21}^{2}=\Gamma_{13}^{3}=\Gamma_{31}^{3}=\frac{1}{r}$} \\
\hline$\Gamma_{33}^{2}=-\sin \theta \cos \theta$ & $\Gamma_{23}^{3}=\Gamma_{32}^{3}=\cot \theta$ \\
\hline
\end{tabular}
metric tensor $g_{\mu \nu}$ in Eq. (22): 
The Ricci curvature tensor given by $R_{\mu \nu}=\partial_{\lambda} \Gamma_{\mu \nu}^{\lambda}-$ $\partial_{v} \Gamma_{\mu \lambda}^{\lambda}+\Gamma_{\mu \nu}^{\rho} \Gamma_{\rho \lambda}^{\lambda}-\Gamma_{\mu \lambda}^{\rho} \Gamma_{\rho \nu}^{\lambda}$. The non-zero components of the Ricci tensor are:

The $t-t$ component is

$$
\begin{gathered}
R_{t t}=R_{00}=-\partial_{0} \Gamma_{01}^{1}-\partial_{0} \Gamma^{2}{ }_{02}-\partial_{0} \Gamma^{3}{ }_{03}-\Gamma_{01}^{1} \Gamma_{10}^{1}{ }_{10} \\
-\Gamma_{02}^{2} \Gamma_{20}^{2}-\Gamma_{03}^{3} \Gamma_{30}^{3} \\
R_{t t}=-3 \partial_{t} \frac{\dot{a}}{a}-3\left(\frac{\dot{a}}{a}\right)^{2}=-3 \frac{\ddot{a} a-\dot{a}^{2}}{a^{2}}-3 \frac{\dot{a}^{2}}{a^{2}}=-3 \frac{\ddot{a}}{a}
\end{gathered}
$$

The $r-r$ component is

$$
\begin{aligned}
& R_{r r}=R_{11}=\partial_{0} \Gamma_{11}^{0}-\partial_{1} \Gamma_{12}^{2}-\partial_{1} \Gamma^{3}{ }_{13}+\Gamma^{0}{ }_{11} \Gamma^{2}{ }_{02}+\Gamma_{11}^{0} \Gamma_{03}^{3} \\
& -\Gamma_{10}^{1} \Gamma_{11}^{0}+\Gamma_{11}^{1} \Gamma_{12}^{2}+\Gamma_{11}^{1} \Gamma_{13}^{3} \\
& R_{r r}=\partial_{t} \frac{a \dot{a}}{c^{2} a_{p}^{2}\left(1-\frac{r^{2}}{r_{p}^{2}}\right)}-2 \partial_{r} \frac{1}{r}+\frac{a \dot{a}}{c^{2} a_{p}^{2}\left(1-\frac{r^{2}}{r_{p}^{2}}\right)} \frac{\dot{a}}{a} \\
& +2 \frac{\mathrm{r}}{r_{p}^{2}\left(1-\frac{r^{2}}{r_{p}^{2}}\right)} \frac{1}{r}-2 \frac{1}{r^{2}} \\
& R_{r r}=\frac{a \ddot{a}}{c^{2} a_{p}{ }^{2}\left(1-\frac{r^{2}}{r_{p}^{2}}\right)}+\frac{\dot{a}^{2}}{c^{2} a_{p}{ }^{2}\left(1-\frac{r^{2}}{r_{p}^{2}}\right)}+\frac{\dot{a}^{2}}{c^{2} a_{p}{ }^{2}\left(1-\frac{r^{2}}{r_{p}^{2}}\right)} \\
& +\frac{2}{r_{p}^{2}\left(1-\frac{r^{2}}{r_{p}^{2}}\right)} \\
& R_{r r}=\frac{\left(\frac{a \ddot{a}}{a_{p}^{2}}+\frac{2 \dot{a}^{2}}{a_{p}^{2}}+\frac{2 c^{2}}{r_{p}^{2}}\right)}{c^{2}\left(1-\frac{r^{2}}{r_{p}^{2}}\right)}
\end{aligned}
$$

The $\theta-\theta$ component is

$$
\begin{aligned}
& R_{\theta \theta}=R_{22}=\partial_{0} \Gamma_{22}^{0}+\partial_{1} \Gamma^{1}{ }_{22}-\partial_{2} \Gamma^{3}{ }_{23}+\Gamma^{0}{ }_{22} \Gamma^{1}{ }_{01}+\Gamma^{0}{ }_{22} \Gamma^{3}{ }_{03} \\
& +\Gamma_{22}^{1} \Gamma_{11}^{1}+\Gamma_{22}^{1} \Gamma^{3}{ }_{13}-\Gamma^{2}{ }_{20} \Gamma^{0}{ }_{22} \\
& -\Gamma^{2}{ }_{21} \Gamma^{1}{ }_{22}-\Gamma_{23}^{3} \Gamma_{32}^{3} \\
& R_{\theta \theta}=\partial_{t} \frac{r^{2} a \dot{a}}{c^{2} a_{p}{ }^{2}}-\partial_{r} \mathrm{r}\left(1-\frac{r^{2}}{r_{p}^{2}}\right)-\partial_{\theta} \cot (\theta)+\frac{r^{2} a \dot{a}}{c^{2} a_{p}^{2}} \frac{\dot{a}}{a} \\
& -\mathrm{r}\left(1-\frac{r^{2}}{r_{p}^{2}}\right) \frac{1}{r}-\cot ^{2}(\theta) \\
& R_{\theta \theta}=\frac{r^{2} a \ddot{a}}{c^{2} a_{p}^{2}}+\frac{r^{2} \dot{a}^{2}}{c^{2} a_{p}^{2}}+\left(3 \frac{r^{2}}{r_{p}^{2}}-1\right)+\csc ^{2}(\theta)+\frac{r^{2} \dot{a}^{2}}{c^{2} a_{p}^{2}} \\
& -\left(1-\frac{r^{2}}{r_{p}^{2}}\right)-\cot ^{2}(\theta) \\
& R_{\theta \theta}=\frac{r^{2} a \ddot{a}}{c^{2} a_{p}^{2}}+2 \frac{r^{2} \dot{a}^{2}}{c^{2} a_{p}^{2}}+\left(2 \frac{r^{2}}{r_{p}^{2}}\right)-1+\csc ^{2}(\theta)-\cot ^{2}(\theta) \\
& R_{\theta \theta}=\frac{r^{2}}{c^{2}}\left(\frac{a \ddot{a}}{a_{p}^{2}}+\frac{2 \dot{a}^{2}}{a_{p}^{2}}+\frac{2 c^{2}}{r_{p}^{2}}\right)
\end{aligned}
$$

The $\phi-\phi$ component is

$$
\begin{aligned}
& R_{\phi \phi}=R_{33}=\partial_{0} \Gamma_{33}^{0}+\partial_{1} \Gamma_{33}^{1}+\partial_{2} \Gamma^{2}{ }_{33}+\Gamma^{0}{ }_{33} \Gamma^{1}{ }_{01}+\Gamma_{33}^{0} \Gamma_{02}^{2} \\
& +\Gamma_{33}^{1} \Gamma_{11}^{1}+\Gamma_{33}^{1} \Gamma_{12}^{2}-\Gamma_{30}^{3} \Gamma_{33}^{0} \\
& -\Gamma_{31}^{3} \Gamma_{33}^{1}-\Gamma_{32}^{3} \Gamma^{2}{ }_{33} \\
& R_{\phi \phi}=R_{33}=\partial_{t} \frac{r^{2} a \dot{a} \sin ^{2} \theta}{c^{2} a_{p}^{2}}-\partial_{r} \mathrm{r} \sin ^{2} \theta\left(1-\frac{r^{2}}{r_{p}^{2}}\right) \\
& -\partial_{\theta} \sin \theta \cos \theta+2 \frac{r^{2} a \dot{a} \sin ^{2} \theta}{c^{2} a_{p}{ }^{2}} \frac{\dot{a}}{a} \\
& -r \sin ^{2} \theta\left(1-\frac{r^{2}}{r_{p}^{2}}\right) \frac{r}{r_{p}^{2}\left(1-\frac{r^{2}}{r_{p}^{2}}\right)} \\
& -\mathrm{r} \sin ^{2} \theta\left(1-\frac{r^{2}}{r_{p}^{2}}\right) \frac{1}{r}-\frac{\dot{a} r^{2} a \dot{a} \sin ^{2} \theta}{c^{2} a_{p}^{2}} \\
& +\mathrm{r} \sin ^{2} \theta\left(1-\frac{r^{2}}{r_{p}^{2}}\right) \frac{1}{r}+\sin \theta \cos \theta \cot \theta \\
& R_{\phi \phi}=R_{33}=\frac{r^{2} a \ddot{a} \sin ^{2} \theta}{c^{2} a_{p}^{2}}+\frac{r^{2} \dot{a}^{2} \sin ^{2} \theta}{c^{2} a_{p}{ }^{2}}-\sin ^{2} \theta\left(1+3 \frac{r^{2}}{r_{p}^{2}}\right) \\
& +\sin ^{2} \theta-\cos ^{2} \theta+\frac{r^{2} \dot{a}^{2} \sin ^{2} \theta}{c^{2} a_{p}{ }^{2}} \\
& -\sin ^{2} \theta\left(\frac{r^{2}}{r_{p}^{2}}\right)+\cos ^{2} \theta \\
& R_{\phi \phi}=R_{33}=\frac{r^{2} a \ddot{a} \sin ^{2} \theta}{c^{2} a_{p}{ }^{2}}+2 \frac{r^{2} \dot{a}^{2} \sin ^{2} \theta}{c^{2} a_{p}{ }^{2}}+2 \sin ^{2} \theta \frac{r^{2}}{r_{p}{ }^{2}} \\
& R_{\phi \phi}=\frac{r^{2} \sin ^{2} \theta}{c^{2}}\left(\frac{a \ddot{a}}{a_{p}^{2}}+\frac{2 \dot{a}^{2}}{a_{p}^{2}}+\frac{2 c^{2}}{r_{p}^{2}}\right)
\end{aligned}
$$

Thirdly, the inverse metric tensor $g^{u v}$ is

$$
g^{u v}=\left(\begin{array}{cccc}
\frac{1}{c^{2}} & 0 & 0 & 0 \\
0 & -\frac{\left(1-\frac{r^{2}}{r_{p}^{2}}\right)}{\left(\frac{a^{2}}{a_{p}^{2}}\right)} & 0 & 0 \\
0 & 0 & \frac{-1}{\left(\frac{a^{2}}{a_{p}^{2}}\right) r^{2}} & 0 \\
0 & 0 & 0 & \frac{-1}{\left(\frac{a^{2}}{a_{p}^{2}}\right) r^{2} \sin ^{2} \theta}
\end{array}\right)
$$

Finally, the Ricci scalar curvature $R=R_{u v} g^{u v}$ which equals the Ricci curvature tensor time the inverse metric tensor as follows

$$
R=R_{u v} g^{u v}=\frac{-6}{c^{2}}\left(\frac{\ddot{a}}{a}+\frac{\dot{a}^{2}}{a^{2}}+\frac{c^{2} a_{p}^{2}}{a^{2} r_{p}^{2}}\right)
$$




\section{Appendix B}

By utilising the conformal time and according to Eq. (14), the conformally transformed metric $\hat{g}_{\mu \nu}$ is $\hat{g}_{u v}=\left(g_{u v}+2 \bar{g}_{u v}\right)=$ $\left(\begin{array}{cccc}\left(1-\frac{2 a_{p}}{a}\right) c^{2} & 0 & 0 & 0 \\ 0 & \frac{\left(\frac{2 a}{a_{p}}-\frac{a^{2}}{a_{p}^{2}}\right)}{\left(1-\frac{r^{2}}{r_{p}^{2}}\right)} & 0 & 0 \\ 0 & 0 & \left(\frac{2 a}{a_{p}}-\frac{a^{2}}{a_{p}^{2}}\right) r^{2} & 0 \\ 0 & 0 & 0 & \left(\frac{2 a}{a_{p}}-\frac{a^{2}}{a_{p}^{2}}\right) r^{2} \sin ^{2} \theta\end{array}\right)$

\begin{tabular}{|c|c|}
\hline$\Gamma_{00}^{0}=\frac{\dot{a}}{a} \frac{a_{p}}{\left(a-2 a_{p}\right)}$ & \\
\hline $\begin{array}{l}\Gamma_{11}^{0} \\
=\frac{a \dot{a}}{c^{2} a_{p}{ }^{2}\left(1-\frac{r^{2}}{r_{p}^{2}}\right)} \frac{\left(a-a_{p}\right)}{\left(a-2 a_{p}\right)}\end{array}$ & $\Gamma_{11}^{1}=\frac{\mathrm{r}}{r_{p}^{2}\left(1-\frac{r^{2}}{r_{p}^{2}}\right)}$ \\
\hline$\Gamma_{22}^{0}=\frac{r^{2} a \dot{a}}{c^{2} a_{p}^{2}} \frac{\left(a-a_{p}\right)}{\left(a-2 a_{p}\right)}$ & $\Gamma_{22}^{1}=-\mathrm{r}\left(1-\frac{r^{2}}{r_{p}^{2}}\right)$ \\
\hline $\begin{array}{l}\Gamma_{33}^{0} \\
=\frac{r^{2} a \dot{a} \sin ^{2} \theta}{c^{2} a_{p}^{2}} \frac{\left(a-a_{p}\right)}{\left(a-2 a_{p}\right)}\end{array}$ & $\Gamma_{33}^{1}=-r \sin ^{2} \theta\left(1-\frac{r^{2}}{r_{p}^{2}}\right)$ \\
\hline \multicolumn{2}{|c|}{$\Gamma_{01}^{1}=\Gamma_{02}^{2}=\Gamma_{03}^{3}=\Gamma_{10}^{1}=\Gamma_{20}^{2}=\Gamma_{30}^{3}=\frac{\dot{a}}{a} \frac{\left(a-a_{p}\right)}{\left(a-2 a_{p}\right)}$} \\
\hline \multicolumn{2}{|l|}{$\Gamma_{12}^{2}=\Gamma_{21}^{2}=\Gamma_{13}^{3}=\Gamma_{31}^{3}=\frac{1}{r}$} \\
\hline$\Gamma_{33}^{2}=-\sin \theta \cos \theta$ & $\Gamma_{23}^{3}=\Gamma_{32}^{3}=\cot \theta$ \\
\hline
\end{tabular}

Christoffel symbols of the second kind for the $\hat{g}_{\mu v}$ metric tensor are solves as given in the table

Data Availability: This study is purely based on mathematical derivations. All derivations are either presented in the main text or in appendices.

\section{References}

Aghanim, N. et al. (2020) 'Planck 2018 results. V. CMB power spectra and likelihoods', Astronomy \& Astrophysics. EDP Sciences, 89. doi: 10.1051/00046361/201936386.

Amendola, L. et al. (2007) 'Conditions for the cosmological viability of $\mathrm{f}(\mathrm{R})$ dark energy models', Physical Review D - Particles, Fields, Gravitation and Cosmology. American Physical Society, 75(8), p. 083504. doi: 10.1103/PhysRevD.75.083504.

Appleby, S. A. and Battye, R. A. (2007) 'Do consistent $\$ F(R) \$$ models mimic General Relativity plus \$Lambda\$?', Physics Letters, Section B: Nuclear, Elementary Particle and High-Energy Physics, 654(1-2), pp. 7-12. doi: 10.1016/j.physletb.2007.08.037.

Bekenstein, J. D. (2007) 'The modified Newtonian dynamics-MOND-and its implications for new physics', Contemporary Physics, 47(6), pp. 387-403. doi: 10.1080/00107510701244055.

Capozziello, S. and Lambiase, G. (2000) 'Higher-order corrections to the effective gravitational action from
Noether symmetry approach', General Relativity and Gravitation. Springer, 32(2), pp. 295-311. doi: 10.1023/A:1001935510837.

Dyer, E. and Hinterbichler, K. (2009) 'Boundary terms, variational principles, and higher derivative modified gravity', Physical Review D - Particles, Fields, Gravitation and Cosmology, 79(2). doi: 10.1103/PhysRevD.79.024028.

Efstathiou, G. (2003) Is the low cosmic microwave background quadrupole a signature of spatial curvature?, Mon. Not. R. Astron. Soc. doi: 10.1046/j.13658711.2003.06940.x.

Ellis, G. F. R. and van Elst, H. (1998) 'Cosmological models (Cargl'\{e\}se lectures 1998)'. Available at: http://arxiv.org/abs/gr-qc/9812046

Garattini, R. (2013) 'Distorting general relativity: Gravity's rainbow and $\mathrm{f}(\mathrm{R})$ theories at work', Journal of Cosmology and Astroparticle Physics. IOP Publishing, 2013(6), p. 017. doi: 10.1088/1475-7516/2013/06/017.

Garriga, J. and Tanaka, T. (1999) 'Gravity in the Randall- 
Sundrum Brane World', Physical Review Letters, 84(13). doi: 10.1103/PhysRevLett.84.2778.

Germani, C. and Sopuerta, C. F. (2002) 'String Inspired Brane World Cosmology', Physical Review Letters. American Physical Society, 88(23), p. 4. doi: 10.1103/PhysRevLett.88.231101.

Kozameh, C. et al. (1985) 'Conformal Einstein spaces', Springer.

Lachì Eze-Rey, M. and Luminet, J.-P. (2003) 'COSMIC TOPOLOGY', arXiv:gr-qc/9605010v2 9 Jan 2003.

Landau, L. D. (1986) Theory of Elasticity, Theory of Elasticity. Elsevier. doi: 10.1016/c2009-0-25521-8.

Mannheim, P. D. (1997) 'Are Galactic Rotation Curves Really Flat?', The Astrophysical Journal. American Astronomical Society, 479(2), pp. 659-664. doi: 10.1086/303933.

Mannheim, P. D. (2001) 'Cosmic Acceleration as the Solution to the Cosmological Constant Problem', The Astrophysical Journal. American Astronomical Society, 561(1), pp. 1-12. doi: 10.1086/323206.

Meneghetti, M. et al. (2020) 'An excess of small-scale gravitational lenses observed in galaxy clusters', Science. American Association for the Advancement of Science, 369(6509), pp. 1347-1351. doi: 10.1126/science.aax5164. Nelson, W. (2010) 'Restricting Fourth Order Gravity via Cosmology', Physical Review D - Particles, Fields, Gravitation and Cosmology, 82(12). doi: 10.1103/PhysRevD.82.124044.

Nojiri, S. and Odintsov, S. D. (2006) 'Introduction to Modified Gravity and Gravitational Alternative for Dark Energy', International Journal of Geometric Methods in Modern Physics, 4(1), pp. 115-145. doi: 10.1142/S0219887807001928.

Nojiri, S. and Odintsov, S. D. (2010) 'Unified cosmic history in modified gravity: from $\mathrm{F}(\mathrm{R})$ theory to Lorentz non-invariant models', Physics Reports, 505(2-4), pp. 59144. doi: 10.1016/j.physrep.2011.04.001.

Pavel Grinfeld (2013) Introduction to Tensor Analysis and the Calculus of Moving Surfaces. Springer.

Penrose, R. (2005) The Road to Reality: A Complete Guide to the Laws of the Universe.

Randall, L. and Sundrum, R. (1999) 'An alternative to compactification', Physical Review Letters. American
Physical Society, 83(23), pp. 4690-4693. doi: 10.1103/PhysRevLett.83.4690.

Rugh, S. E. and Zinkernagel, H. (2000) 'The Quantum Vacuum and the Cosmological Constant Problem', Studies in History and Philosophy of Science Part B - Studies in History and Philosophy of Modern Physics. Elsevier Ltd, 33(4), pp. 663-705.

Ryden, B. (2006) Introduction to Cosmology. San Francisco, CA, USA: Addison Wesley, ISBN 0-80538912-1.

S. M. Carroll (2003) 'Spacetime and Geometry: An Introduction to General Relativity'. doi: 10.1088/02649381/22/20/B01.

Straub, W. O. (2006) Simple Derivation of the Weyl Conformal Tensor, academia.edu.

Straumann, N. (2013) 'General Relativity (Graduate Texts in Physics)', in Springer. Springer.

Umetsu, K. (2020) 'Cluster-galaxy weak lensing', Astronomy and Astrophysics Review. Springer Science and Business Media Deutschland GmbH, pp. 1-106. doi: 10.1007/s00159-020-00129-w.

Di Valentino, E., Melchiorri, A. and Silk, J. (2020) 'Planck evidence for a closed Universe and a possible crisis for cosmology', Nature Astronomy. Nature Research, 4(2), pp. 196-203. doi: 10.1038/s41550-019-0906-9.

Vikman, A. (2005) 'Can dark energy evolve to the phantom?', Physical Review D - Particles, Fields, Gravitation and Cosmology. American Physical Society, 71(2), p. 023515. doi: 10.1103/PhysRevD.71.023515.

Vilkovisky, G. A. (1992) 'Effective action in quantum gravity', Classical and Quantum Gravity. IOP Publishing, 9(4), pp. 895-903. doi: 10.1088/0264-9381/9/4/008. 